\title{
Climate negotiations How to break the impasse and deliver
}

\author{
Juan Zak and Myung Kyoon Lee \\ Book chapter in Post-2020 Climate Change Regime Formation \\ 2013, Routledge
}

\begin{abstract}
The current climate regime has proven largely insufficient to stabilize the global climate system. The root of the shortfall is in the system adopted to reduce emissions: meager binding targets for developed countries based on absolute 1990 levels. The issue of how to adopt more realistic and equitable allocation of emissions reduction targets has been a permanent impasse in the international climate negotiations.
\end{abstract}

There are two essential considerations to break the impasse and deliver an effective and equitable post-Kyoto climate regime:

1) The emissions rights implicit in the Kyoto Protocol are ultimately inequitable. If percapita global emissions must be halved on an equitable basis to stabilize climate, then most developing countries have always been under this sustainable target.

2) It is richer individuals globally, and not developed countries per se, who have contributed most to global warming. Equity should transcend national borders: the poor in developed countries have the same rights to emit as anybody else; conversely, richer individuals in developing countries have the same obligation to reduce emissions as their counterparts in developed countries.

To be equitable, any new climate regime should be based on per-capita emissions. To be effective, the new regime should limit emissions directly at the root -the consumers- as new evidence emerges that renewables, nuclear fission and technologies per se in the near future cannot support current consumption patterns in a sustainable way.

While every individual in a country should receive the same equitable emissions allowance, flexibility in fitting different needs should be provided by national trading of unused allowances. Every signatory country should receive an equitable share of allowances proportional to its population. While trading between countries should provide flexible distribution of allocated allowances, the total amount of allowances globally should remain constant as per the agreed global reduction target.

At first glance, a system of per-capita emissions allowances could look unfeasible. Monetizing emissions allowances and prioritizing tracked variables should make the system practicable. A concerted international effort could also contribute to making the system operational by less developed countries. 
The implementation difficulties of a per-capita allowance system should be assessed in comparison to the difficulties in implementing a post-2012 climate regime built on the Kyoto architecture. Successfully implementing the extended Kyoto flexibility mechanisms or their successors is seemingly more difficult than implementing the more straightforward and transparent per-capita allowance system.

This chapter provides an early outline of how a system of per-capita emissions allowances would work. More research is needed on the detailed design and implementation issues of a new climate regime based on this system.

The system of per-capita GHG emissions allowances proposed in this chapter could be eventually extended to other natural resources subject to unsustainable consumption, at the expense of the environment and future generations. 